\title{
CD73 expression in myeloid-derived suppressor cells is correlated with clinical stages in head and neck squamous cell carcinomas
}

\author{
Weihui Zheng ${ }^{1,2 \#}$, Ying Zhu ${ }^{1,2 \#}$, Xiaolong Chen ${ }^{3}$, Jianqiang Zhao ${ }^{1,2}$ \\ ${ }^{1}$ The Cancer Hospital of the University of Chinese Academy of Sciences (Zhejiang Cancer Hospital), Institute of Basic Medicine and Cancer (IBMC), \\ Chinese Academy of Sciences, Hangzhou, China; ${ }^{2}$ Key Laboratory of Head \& Neck Cancer Translational Research of Zhejiang Province, Hangzhou, \\ China; ${ }^{3}$ NanoDrug Platform, Zhejiang California International NanoSystems Institute, Zhejiang University, Hangzhou, China \\ Contributions: (I) Conception and design: J Zhao; (II) Administrative support: J Zhao; (III) Provision of study materials or patients: J Zhao, Y Zhu; (IV) \\ Collection and assembly of data: W Zheng, Y Zhu; (V) Data analysis and interpretation: W Zheng, X Chen; (VI) Manuscript writing: All authors; (VII) \\ Final approval of manuscript: All authors. \\ "These authors contributed equally to this work. \\ Correspondence to: Jianqiang Zhao. The Cancer Hospital of the University of Chinese Academy of Sciences (Zhejiang Cancer Hospital), Institute of Basic \\ Medicine and Cancer (IBMC), Chinese Academy of Sciences, No. 1, East Banshan Road, Hangzhou 310022, China. Email: zhaojq@zjcc.org.cn.
}

Background: Ecto-5'-nucleotidase (cluster of differentiation 73/CD73) is an ectonucleotidase that is being evaluated as a biomarker for the diagnosis and prognosis of various types of cancer. However, the clinicopathological relationship between CD73 expression in monocytic MDSCs (M-MDSCs) and polymorphonuclear MDSC (PMN-MDSCs) in head and neck squamous cell carcinomas (HNSCCs) is not clear. Understanding the phenotypic and functional characteristics of human $\mathrm{CD} 73^{+} \mathrm{MDSCs}$ in the tumor microenvironment could help elucidate the roles of these cells in the ontogeny, spread, and treatment of solid cancer.

Methods: In the present study, we first analyzed the expression percentage of human M-MDSCs and PMN-MDSCs subsets circulating in peripheral blood of patients with head and neck tumors originated in nasopharynx, oropharynx, oropharynx and larynx. To identify the correlation between phenotypic characteristics of MDSCs and clinical stages in HNSCC, we extended the study by analyzing the percentage, CD73 phenotype and immunosuppressive function of MDSCs and the correlation with the clinical parameters. Moreover, we compare the functions of both M-MDSCs and PMN-MDSCs blunts T-cell function in an ectonucleotidase-dependent manner.

Results: Our study revealed that PMN-MDSCs were significantly increased in HNSCC patients, contributing to MDSC-mediated T cell immune suppression. Our results indicated that PMN-MDSCs comprised the majority of MDSCs participating in anticancer immunosuppression. The increase in PMNMDSCs was directly correlated with the clinical stages of HNSCC. Levels of CD73 were increased in PMN-MDSCs and were correlated with the clinical stages of HNSCC. The ectonucleotidase inhibitor adenosine 5 '-( $\alpha, \beta$-methylene)diphosphate (APCP) decreased its suppression towards $\mathrm{T}$ cell proliferation. Ectonucleotidase inhibitors are promising candidates for the treatment of HNSCC.

Conclusions: These studies demonstrate the expansion of PMN-MDSCs correlated with expression of CD73 and increasing clinical stages in HNSCC. These CD73 ${ }^{+} \mathrm{PMN}-\mathrm{MDSC}$ contributes to T cell immune suppression activity in HNSCC patients. Using ectonucleotidase inhibitors is a promising rationale for PMN-MDSCs in future clinical development of immunotherapy in human HNSCC cancer.

Keywords: Head and neck squamous cell carcinoma (HNSCC); polymorphonuclear myeloid-derived suppressor cells (PMN-MDSCs); CD73; tumor immunoescape

Submitted Apr 23, 2021. Accepted for publication Jul 06, 2021.

doi: 10.21037/atm-21-2589

View this article at: https://dx.doi.org/10.21037/atm-21-2589 


\section{Introduction}

Head and neck squamous cell carcinoma (HNSCC) affects several regions of the human throat. By incidence, it is one of the most common cancers worldwide and has resulted in 14,620 deaths in United States $(1,2)$. Currently, a combination of surgery, chemotherapy, and radiotherapy is used for the treatment of HNSCC patients. Despite the multimodality approach to its treatment, the survival rate of HNSCC patients has not improved $(3,4)$. As a promising adjunct treatment, immunotherapy may help reduce longterm toxicities of the standard treatments and improve survival rates.

An increase in myeloid-derived suppressor cells (MDSCs) is caused by HNSCC, which results in malfunction of $\mathrm{T}$ cells and dendritic cells. The increase in levels of immunosuppression cells has been widely investigated (5-7). MDSCs are of a heterogeneous population of immature myeloid cells consisting of immature dendritic cell, macrophage, and granulocyte progenitors that blocked at several stages of differentiation $(8,9)$. There are 2 major subtypes of MDSCs: monocytic MDSCs (M-MDSCs) and polymorphonuclear (PMN-MDSCs) which typically express the common myeloid markers, CD33 and CD11b, but lack markers of mature myeloid cells, such as $\operatorname{HLA}-\mathrm{DR}(10,11)$. Various factors of different cancer types enable a high degree of plasticity in the phenotypes of MDSCs present in cancer patients (12-15).

High levels of ecto-5'-nucleotidase (CD73) are found in the MDSCs in patients with various cancers, promoting the progression of cancer by converting adenosine triphosphate (ATP) and adenosine diphosphate (ADP) into adenosine (16). Adenosine inhibits effector $T$ cell function and enhances regulatory $\mathrm{T}$ cell function, which results in the reduction of $\mathrm{T}$ cell proliferation and increases cancer growth $(17,18)$. Increased CD73 expression occurs in numerous tumor types, and is not only correlated with the clinical stage of the cancers $(19,20)$ but also serves as a prognostic and diagnostic marker in malignancies, including HNSCC, prostate cancer, and ovarian cancer (21-23). In HNSCC, CD73 helps mediate the immune escape mechanisms of cancer, promoting tumor growth and progression $(24,25)$. Investigation of the functional characteristics and phenotypic characteristics of MDSCs may reveal the progression of HNSCC, how it spreads, and how to treat it (16). Although the expression of CD73 in MDSCs in several cancers such as ovarian and non-small cell lung cancer have been reported $(23,26)$. However, there is little information about CD73 expression in MDSC subsets from HNSCC and how these cells are participating in anticancer immunosuppression, especially the relationship between suppression of PMN-MDSCs subtype towards $\mathrm{T}$ cell suppression is unclear. Here, the percentage, CD73 phenotype and immunosuppression down-regulation of human M-MDSCs and PMN-MDSCs subsets circulating in peripheral blood of patients with head and neck tumors were investigated in regard to HNSCC severity and clinicopathological characteristics. We present the following article in accordance with the MDAR reporting checklist (available at https://dx.doi. org/10.21037/atm-21-2589).

\section{Methods}

\section{Participant recruitment}

A total of 53 test patients with HCSCC [ 42 males and 11 females; mean age, 57; standard deviation (SD), 12 years; range, 28 to 78 years] and 12 control (non-cancer) patients were recruited at the Zhejiang Cancer Hospital between 1 January 2017 and 31 December 2019. All test patients were pathologically confirmed with HNSCC and had not undergone any single or multimodal treatments before phlebotomy. The cohort included 23 stage I patients, 10 stage II patients, and 20 stage III patients. A total of 12 carcinomas had originated in the oral cavity $(\mathrm{n}=12), 28$ were from the larynx, and 13 from the oropharynx. Approval of the study was granted by the Institutional Review Board of the Zhejiang Cancer Hospital. Signed informed consent forms were provided by all participants. The study was conducted in accordance with the Declaration of Helsinki (as revised in 2013). Clinical characteristics of the test and control participants were determined and recorded (Table 1).

\section{Flow cytometry analysis of MDSC subsets}

Cell analysis was performed using an ACEA NovoCyte Flow Cytometer (Agilent, Santa Clara, CA, USA) to establish the percentages of M-MDSCs and PMN-MDSCs in $\mathrm{CD} 11 \mathrm{~b}^{+} \mathrm{CD} 33^{+}$MDSCs. Fluorescence-conjugated monoclonal antibodies were purchased from BioLegend (San Diego, CA, USA). Gating was restricted based on the cell properties in the forward and side scatters. Linear scaling was used for plotting the scatters and at least $1 \times 10^{5}$ cells were assessed. The $\mathrm{CD} 11 \mathrm{~b}^{+} \mathrm{CD} 33^{+}$myeloidderived cell populations were first gated using FITC-anti- 
Table 1 Clinicopathological characteristics of the patients enrolled in this study $(\mathrm{n}=53)$

\begin{tabular}{|c|c|}
\hline Characteristic & Value \\
\hline Total No. patients (male/female) & $53(42 / 11)$ \\
\hline Age, mean [range] (years) & $57.3[28-78]$ \\
\hline \multicolumn{2}{|l|}{ Tumor site } \\
\hline Oral cavity & 12 \\
\hline Oropharynx & 13 \\
\hline Larynx & 28 \\
\hline \multicolumn{2}{|l|}{ Tumor differentiation, n (\%) } \\
\hline G1 & $38(71.7)$ \\
\hline G2 & $9(17.0)$ \\
\hline G3 & $6(11.3)$ \\
\hline \multicolumn{2}{|l|}{ T stage, $\mathrm{n}(\%)$} \\
\hline $\mathrm{T} 1$ & $17(32.1)$ \\
\hline $\mathrm{T} 2$ & $21(39.7)$ \\
\hline T3 & $6(11.3)$ \\
\hline $\mathrm{T} 4$ & 9 (16.9) \\
\hline \multicolumn{2}{|l|}{ Clinical stage, n (\%) } \\
\hline I & $23(43.4)$ \\
\hline II & $10(18.9)$ \\
\hline III & $20(37.7)$ \\
\hline
\end{tabular}

CD33 (Cat\#303303) and PE-anti-CD11b (Cat\#393111) antibodies. Antibodies of PE-cy7-CD14 (Cat\# 367111) and APC-HLA-DR (Cat\# 327021) were used to stain the M-MDSCs and PE-cy7-CD14 and APC-CD15 (Cat\#301907) antibodies were used to stain the PMNMDSCs. The expression levels of CD73 in MDSCs were assessed with PerCP-Cy5.5-CD73 (Cat\#344013) antibodies. The controls were isotype-matched antibodies. Data analysis was carried out using NovoExpress software (Agilent, USA).

\section{Cell isolation and sorting}

Density gradient centrifugation was performed using the Ficoll-Hypaque separation method to purify peripheral blood mononuclear cells (PBMCs). Briefly, participant blood samples $(30 \mathrm{~mL})$ were drawn into heparinized blood collection tubes and diluted with 1:1 volume $1 \times$ phosphate-buffered saline (PBS). The cells were added to a Ficoll-Hypaque column (GE Healthcare, Chicago, IL, USA) and centrifuged. The PBMCs were collected and washed twice with $1 \times$ PBS buffer. Trypan blue dye was used to assess cell viability.

Using a magnetic-activated cell sorting (MACS) magnetic sorting system (Miltenyi Biotec, Bergisch Gladbach, Germany), and the CD14 $4^{+} \mathrm{CD} 11 \mathrm{~b}^{+}$(M-MDSCs) and CD14-CD11 b ${ }^{+}$(PMN-MDSCs) cells were enriched. The phenotype and purity of the cells in each subset were confirmed: $\mathrm{M}-\mathrm{MDSCs}\left(\mathrm{CD} 14^{+} \mathrm{CD} 11 \mathrm{~b}^{+}\right)$were $>88 \%$ pure and PMN-MDSCs (CD14- CD11 ${ }^{+}$) were $>87 \%$ pure. A CD73 ${ }^{+}$MDSC subset was isolated using BD FACSMelody ${ }^{\mathrm{TM}}$ Cell Sorter (Becton, Dickinson, and Co., Franklin Lakes, NJ, USA) with a purity $>90 \%$. We also isolated $\mathrm{CD}^{+}{ }^{+} \mathrm{T}$ cells using $\mathrm{CD}^{+}{ }^{+}$immunomagnetic beads with a purity of $>95 \%$.

\section{In vitro T cell suppression assay}

Carboxyfluorescein succinimidyl ester (CFSE) at $2 \mathrm{mM}$ (Invitrogen Molecular Probes, Carlsbad, CA, USA) was used to label purified $\mathrm{T}$ cells. Approximately $1 \times 10^{5}$ labeled T cells were incubated at 1:2 and 1:4 ratios with $\mathrm{CD} 73^{+}$ MDSC subsets including $1 \mu \mathrm{g} / \mathrm{mL}$ each of anti-CD3/antiCD28 antibodies. To examine CD73 suppression, CFSE$\mathrm{CD}^{+} \mathrm{T}$ cells were added to MDSCs pretreated with 200 $\mathrm{mM}$ adenosine $5^{\prime}-(\alpha, \beta$-methylene)diphosphate (APCP) (AMP, Sigma-Aldrich, St. Louis, MO, USA) and incubated for $2 \mathrm{~h}$ at $37^{\circ} \mathrm{C}$. After $72 \mathrm{~h}, \mathrm{CD} 3^{+} \mathrm{CD} 28^{+} \mathrm{T}$ cell proliferation was determined using flow cytometry. Enzymelinked immunosorbent assay (ELISA) (R\&D Systems, Minneapolis, MN, USA) was used to analyze supernatants for interferon- $\gamma(\mathrm{IFN}-\gamma)$ levels.

\section{Statistical analysis}

The software GraphPad Prism 8.0 (GraphPad Software, La Jolla, CA, USA) was used to perform statistical analysis. Either the Student's $t$-test or the Mann-Whitney U-test was used to analyze the flow cytometry data. An analysis of correlations was assessed using Spearman's correlation coefficient. Statistical significance was set to $\mathrm{P}<0.05$.

\section{Results}

PMN-MDSCs are bighly enriched in patients with HNSCC and associated with clinical progress

The PMN-MDSCs had a CD $11 \mathrm{~b}^{+} \mathrm{CD} 33^{+} \mathrm{CD} 15^{+} \mathrm{CD} 14^{-}$ 


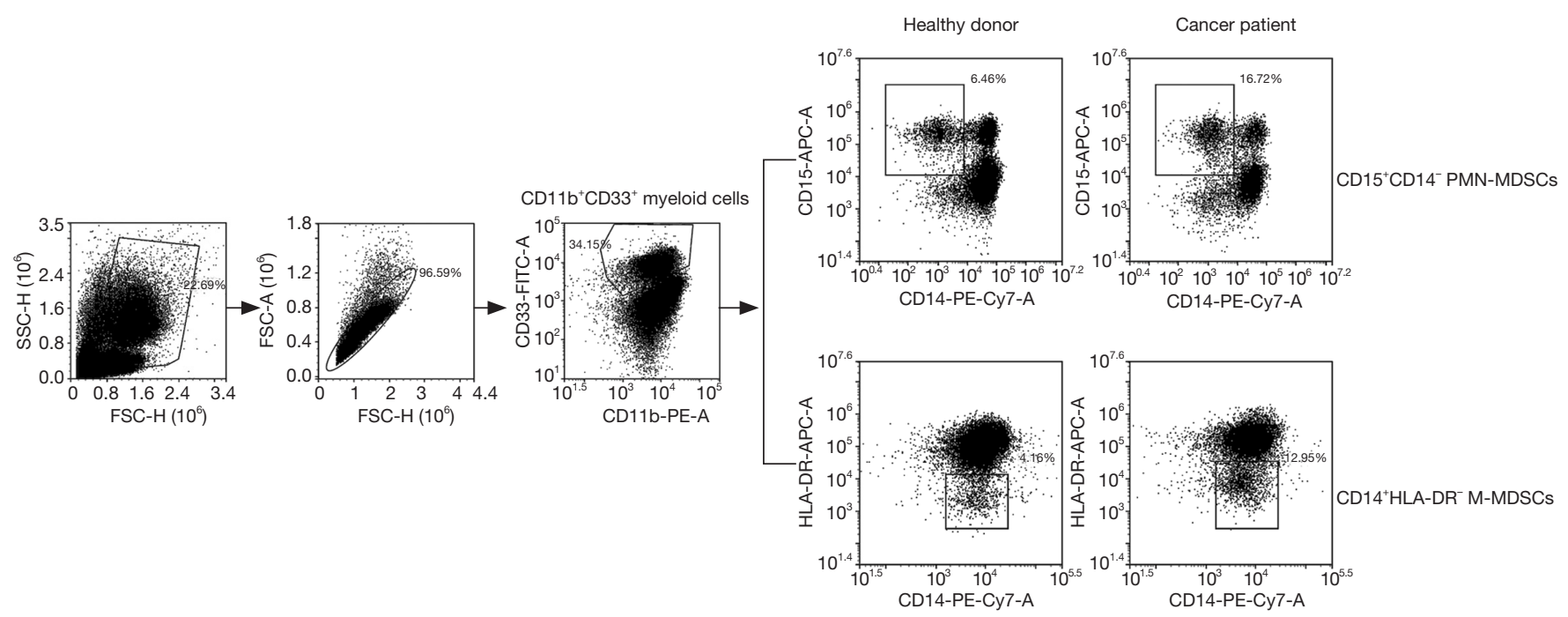

Figure 1 Graphical representation of MDSCs definition by flow cytometry. To determine the percentage of MDSC subsets in patients,

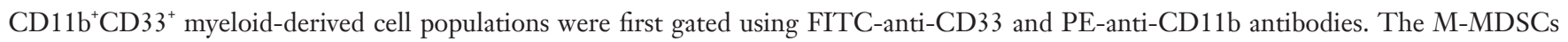
were stained with PE-cy7-CD14 and APC-HLA-DR antibodies while PE-cy7-CD14 and APC-CD15 antibodies for PMN-MDSCs. MDSCs, myeloid-derived suppressor cells; M-MDSCs, monocytic myeloid-derived suppressor cells; PMN-MDSCs, polymorphonuclear myeloid-derived suppressor cells.

phenotype and M-MDSCs had a CD11 $\mathrm{b}^{+} \mathrm{CD} 33^{+} \mathrm{CD} 14^{+} \mathrm{HLA}-$ $\mathrm{DR}^{-}$phenotype. Representative flow cytometry results are shown in Figure 1. The M-MDSCs were significantly elevated in HNSCC patients (mean, 9.01\%; $\mathrm{P}<0.001$ ) relative to healthy donors (mean $2.63 \%$ ) (Figure $2 A$ ). The MDSC subtypes were compared with clinical cancer stages, tumor, node, metastasis (TNM) stages, and tumor differentiation. And the levels of M-MDSCs was observed a significant positive correlation between healthy donors (mean, 2.06\%) and patients at stage I (mean, 7.55\%; $\mathrm{P}<0.001$ ), stage II (mean, 9.97\%; $\mathrm{P}<0.001$ ), and stage III (mean, $10.17 \% ; \mathrm{P}<0.001$ ) (Figure $2 B$ ). Stage III HNSCC patients demonstrated a significantly higher percentage of M-MDSCs compared with those at stages I/II $(\mathrm{P}=0.0149)$. However, no significant associations of M-MDSCs were observed with TMN stage or tumor differentiation (Figure $2 C, D$ ) due to the large variations in these samples.

The PMN-MDSCs were also significantly increased (mean 13.2; $\mathrm{P}<0.001)$ compared with those in normal donors (mean $1.93 \%$ ) (Figure $3 A$ ). Analysis of the percentage of PMN-MDSCs at different clinical stages revealed similar results. A significant positive correlation of PMN-MDSC levels was observed between healthy donors (mean, 1.88\%) and patients at stage I (mean, 7.55\%; $\mathrm{P}<0.001$ ), stage II (mean, $10.55 \% ; \mathrm{P}<0.001$ ), and stage III (mean, 10.28\%; $\mathrm{P}<0.001$ ) (Figure 3B). Direct correlations among PMN-MDSC percentages and clinical cancer stages were determined for stage I vs. stage II $(\mathrm{P}=0.0364)$, stage I vs. stage III $(\mathrm{P}<0.001)$, and stage II vs. stage III $(\mathrm{P}=0.0167)$. But no significant associations of $\mathrm{PMN}-\mathrm{MDSC}$ were observed with TMN stage or tumor differentiation (Figure 3C,D).

\section{CD73 expression in PMN-MDSCs correlates with clinical cancer stage}

We investigated MDSCs from patients and healthy volunteers in order to determine the role of CD73 in tumor immunopathology. In HNSCC patients, levels of M-MDSCs (mean, $8.19 \% ; \mathrm{P}<0.001$ ) and PMN-MDSCs $(10.76 \% ; \mathrm{P}<0.001)$ had significantly higher levels of CD73 compared to those of healthy donors (mean, $2.16 \%$ and $1.19 \%$, respectively) (Figure $4 A, B)$. However, no correlation was observed between $\mathrm{CD} 73^{+} \mathrm{M}-\mathrm{MDSC}$ and clinical stage (Figure $4 C$ ). The $\mathrm{CD} 73^{+} \mathrm{PMN}-\mathrm{MDSCs}$ demonstrated significant correlations with stage I (mean, 7.74\%), stage II (mean, 10.32\%), and stage III (mean, 14.38\%); stage I vs. III $\mathrm{P}<0.001$, stage II vs. III, $\mathrm{P}=0.029$, respectively) (Figure $4 D$ ). No associations were observed between the percentages of MDSCs and TNM stage or tumor differentiation (data not shown). 
A

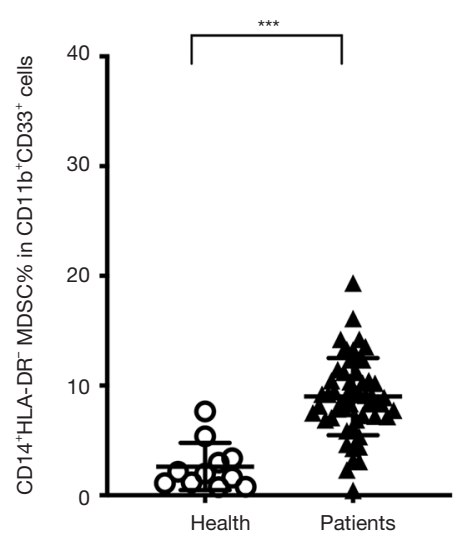

C

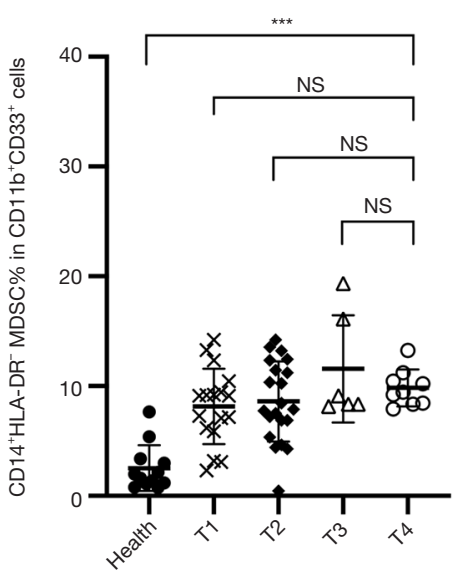

B

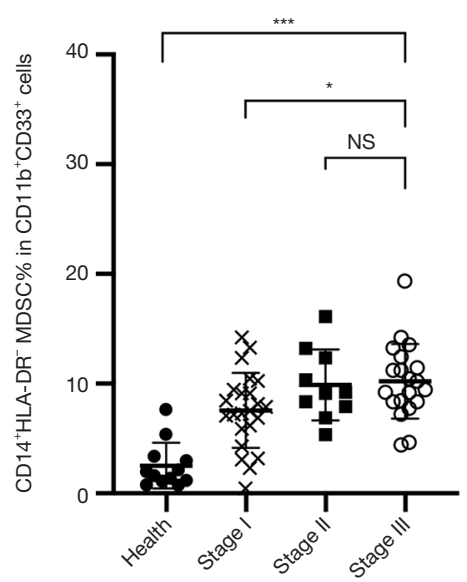

D

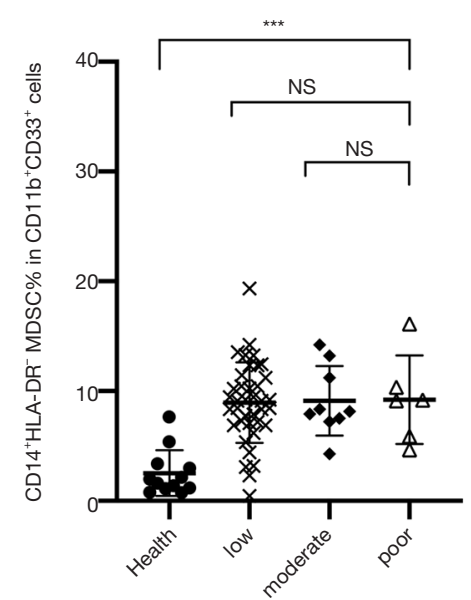

Figure 2 The frequency of M-MDSCs in peripheral blood of cancer patients and healthy donors. (A) CD14 HLA-DR ${ }^{-}$M-type MDSCs were significantly elevated in patients relative to healthy donors (mean $9.01 \% v s .2 .63 \%, \mathrm{P}<0.0001$ ). (B) A significant positive correlation was observed between healthy donors and patients with different stage (mean $2.06 \%$ vs. $7.55 \%, 9.97 \%$ and $10.17 \%$ for healthy vs. stage I, II and III; $\mathrm{P}<0.0001, \mathrm{P}<0.0001, \mathrm{P}<0.0001$ respectively). No correlation between the M-MDSCs and TNM stage (C) or tumor differentiation (D) stage. * indicates a $\mathrm{P}$ value of $<0.05$; ${ }^{* * *}$ indicates a $\mathrm{P}$ value of $<0.0001$. MDSCs, myeloid-derived suppressor cells; M-MDSCs, monocytic myeloid-derived suppressor cells; TNM, tumor, node, metastasis; Health, healthy volunteers; Patients, HNSCC patients.

\section{CD73 activity contributes to PMN-MDSC-mediated T cell immune suppression}

To understand the suppression effect of CD73 in MDSCs on $\mathrm{T}$ cell activation, CFSE-labeled $\mathrm{CD}^{+} \mathrm{T}$ cells were co-cultured with $\mathrm{CD} 73^{+} \mathrm{M}$ - or PMN-MDSCs both in the absence and presence of the CD73 inhibitor APCP. Inhibition of the division of $\mathrm{T}$ cells was performed in a dose-dependent manner using CD $73^{+}$PMN-MDSCs (Figure 5A). The $\mathrm{CD} 73^{+} \mathrm{M}$-MDSCs also demonstrated dose-dependent inhibition of autologous $\mathrm{T}$ cell division, but to a lesser extent (Figure 5A). Similar results were obtained using an IFN- $\gamma$ ELISA assay (Figure $5 B$ ). Suppression of T cells by $\mathrm{CD} 73^{+} \mathrm{PMN}-\mathrm{MDSC}$ was attenuated by APCP; however, there was much less attenuation of M-MDSC activity by APCP. The expression of IFN- $\gamma$ in T cells was inhibited by $\mathrm{CD} 73^{+}$MDSCs. However, IFN- $\gamma$ expression was restored by the addition of APCP. These results indicate that CD73 activity contributes to MDSC-mediated immune suppression.

\section{Discussion}

The progression of various cancers, including HNSCC, is promoted by MDSCs, which consist of immunosuppressive progenitors and immature cells. However, the subsets of 
A

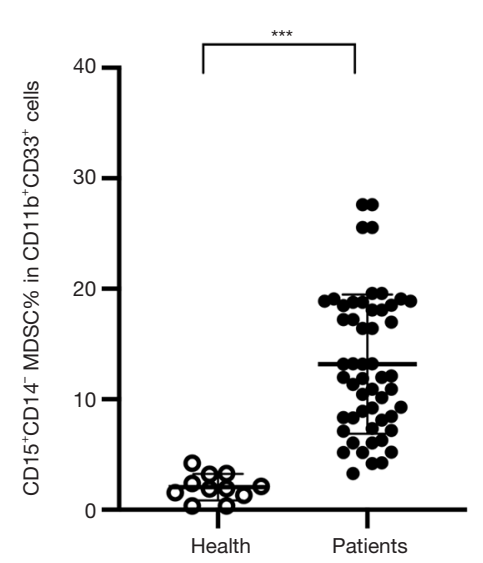

C

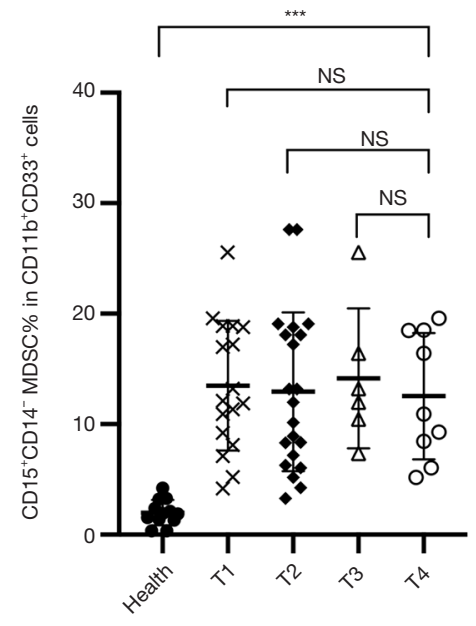

B

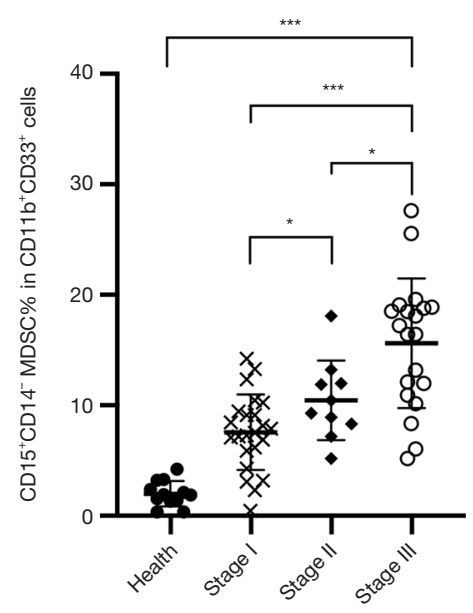

D

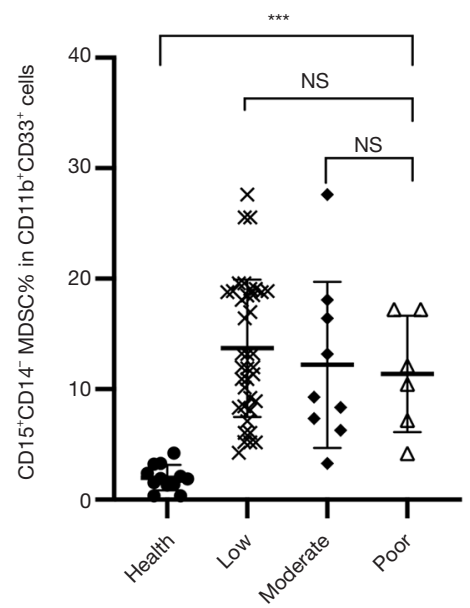

Figure 3 The frequency of PMN-MDSCs in the peripheral blood obtained from cancer patients and healthy donors. (A) CD15 $5^{+} \mathrm{CD} 14^{-}$ PMN-MDSCs were also significantly increased compared with those in normal donors (mean $13.19 \%$ vs. $1.93 \%, \mathrm{P}<0.0001$ ). (B) Compared to normal donors, the number of PMN-MDSCs in cancer patients was (mean $1.88 \%$ vs. $7.55 \%, 10.28 \%$, and $15.47 \%$ for healthy $v s$. stage I, II, and III; $\mathrm{P}<0.0001, \mathrm{P}<0.0001, \mathrm{P}<0.0001$, respectively). A direct correlation between clinical cancer stage and PMN-MDSCs percentage was observed for stage I vs. II ( $\mathrm{P}=0.0364)$, stage I vs. III $(\mathrm{P}<0.0001)$ and stage II $v s$. III $(\mathrm{P}=0.0167)$, respectively. No correlation was observed between the PMN-MDSCs and a TNM stage (C) or tumor differentiation (D) stage. * indicates a $\mathrm{P}$ value of $<0.05$; *** indicates a $\mathrm{P}$ value of $<0.0001$. PMN-MDSCs, polymorphonuclear myeloid-derived suppressor cells; TNM, tumor, node, metastasis; Health, healthy volunteers; Patients, HNSCC patients.

myeloid cells have been poorly characterized (11). High levels of the ectonucleotidase CD73 can be expressed by MDSCs, resulting in the production of high levels of adenosine, which in turn promotes the progression of various cancers (22,24,27-30). It appears that MDSCs play a crucial role in regulating antitumor immune responses in HNSCC (31). Our study found that CD $14^{+}$HLA$\mathrm{DR}^{-} \mathrm{M}-\mathrm{MDSCs}$ and $\mathrm{CD} 15^{+} \mathrm{CD} 14^{-} \mathrm{PMN}-\mathrm{MDSCs}$ in

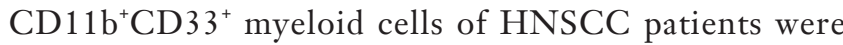
significantly increased compared to those of healthy donors, which was consistent with prior observations $(8,9,32,33)$. No correlation between the percentages of M-MDSCs and PMN-MDSCs was observed, suggesting that the accumulation of these subtypes was independent of each other in HNSCC patients. The percentage of M-MDSCs was not correlated with tumor stage, TNM stage, or tumor 
A

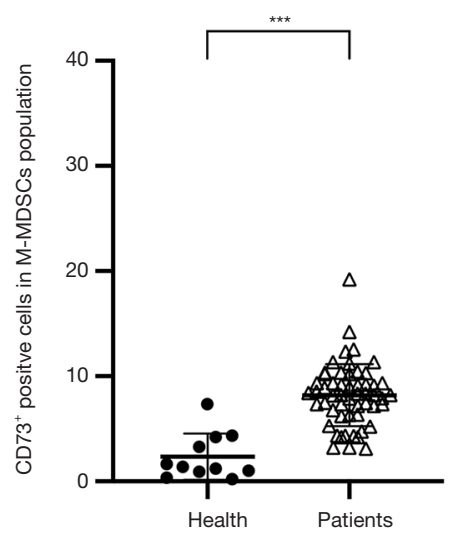

C

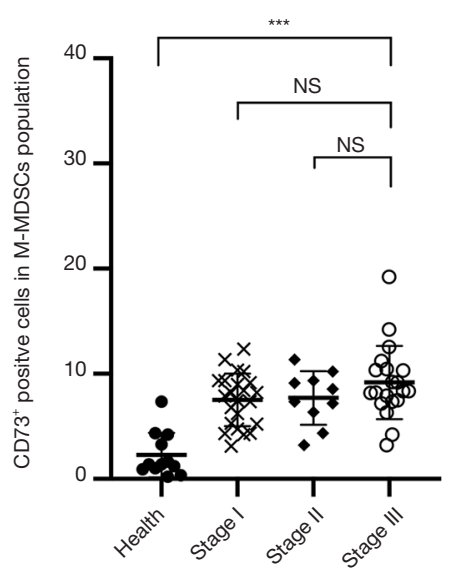

B

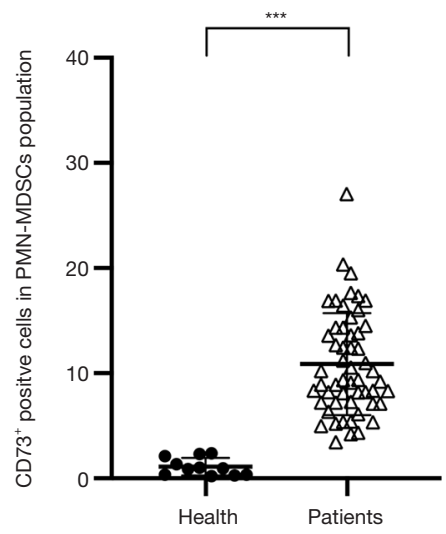

D

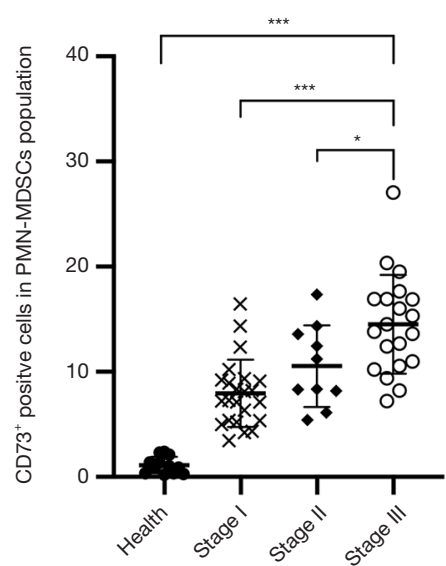

Figure 4 The percentage of CD73 expression on peripheral blood MDSCs. Both M-MDSCs (A) and PMN-MDSCs (B) from HNSCC patients expressed significant high level of $\mathrm{CD} 73$ than those from healthy donors with mean $8.19 \%$ vs. $2.16 \%(\mathrm{P}<0.0001)$ and $10.76 \% v s$. $1.19 \%(\mathrm{P}<0.0001)$. (C) No correlation was observed between CD73 ${ }^{+}$M-MDSCs and the clinical stage. (D) The CD73 $3^{+}$PMN-MDSCs was specifically correlated with the clinical stage (mean $7.74 \%, 10.32 \%$ and $14.38 \%$ for stage I, II and III; stage I $v s$. III P $<0.0001$, stage II vs. III, $\mathrm{P}=0.029$ respectively). * indicates a $\mathrm{P}$ value of $<0.05 ;{ }^{* * *}$ indicates a $\mathrm{P}$ value of $<0.0001$. MDSCs, myeloid-derived suppressor cells; PMN-MDSCs, polymorphonuclear myeloid-derived suppressor cells; HNSCC, head and neck squamous cell carcinoma; Health, healthy volunteers; Patients, HNSCC patients.

differentiation. There was a significant correlation between PMN-MDSC percentages and tumor stage, but not with TNM stage or tumor differentiation. A variety of studies have characterized MDSCs as LinHLA-DR ${ }^{-} \mathrm{CD} 33^{+} \mathrm{CD} 11 \mathrm{~b}^{+}$. Correlations between LinHLA-DR-CD $33^{+} \mathrm{CD} 11 \mathrm{~b}^{+}$MDSCs and tumor stages in HNSCC and various other tumors have been reported (9). This correlation is useful to characterize the overall changes in myeloid cells in cancer patients. However, it does not accurately and unequivocally define MDSCs subsets. Other studies have shown that the levels of CD14+HLA$\mathrm{DR}^{-} \mathrm{M}-\mathrm{MDSC}$ cells do not correlate with the stages of
HNSCC (33). However, correlations between the percentage of M-MDSCs and the clinical status in tumor-infiltrating mononuclear cells are significant. Proinflammatory cytokines and angiogenic factors can also recruit M-MDSCs cells to tumor sites (31). Our results showed that significant differences were observed only between healthy patients and those with advanced tumors. Stages I-III of HNSCC showed moderately increased percentages without significant differences. We did not observe significant correlations between M- and PMN-MDSC percentages and TMN stage or tumor differentiation, which may have been because the 
A

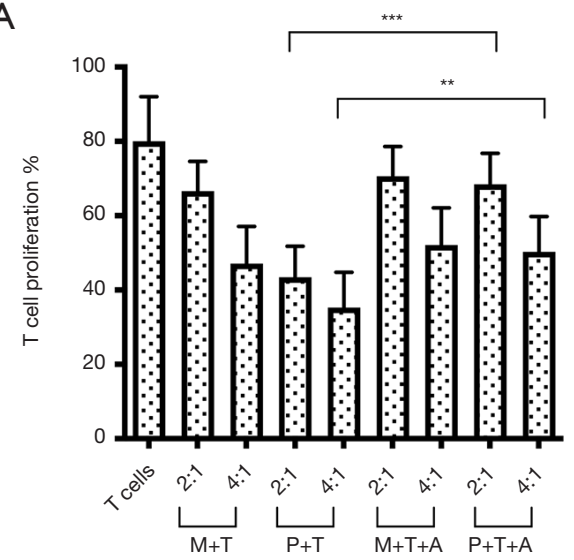

B

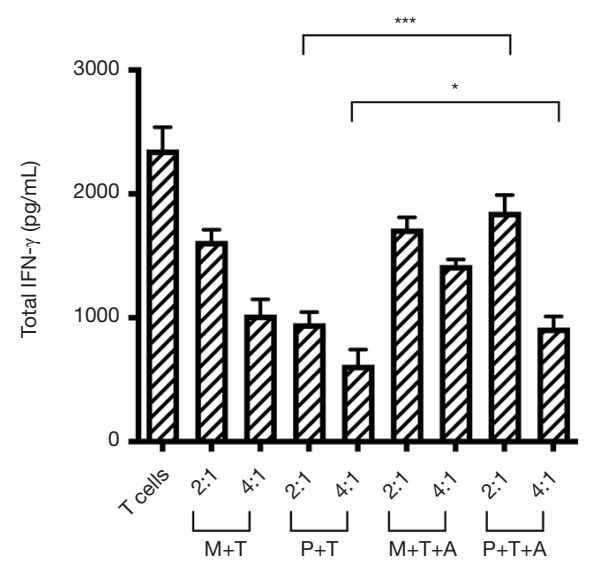

Figure 5 Isolated $\mathrm{CD}^{+} 3^{+} \mathrm{MDSC}$ from cancer patients inhibits $\mathrm{CD}^{+}$T-cell proliferation. (A) Purified CD ${ }^{+} \mathrm{T}$ cells $(\mathrm{T})$ were stimulated by anti-CD3/anti-CD28 in the presence of indicated MDSCs subsets (M for M-MDSCs and P for PMN-MDSCs) from peripheral blood of HNSCC patients at M:T ratios of 2:1 and 4:1 for $3 \mathrm{~d}(\mathrm{n}=4)$. (B) Purified MDSCs subsets were incubated at a ratio of 2:1 or 4:1 with autologous $\mathrm{CD}^{+} \mathrm{T}$ cells in the presence of CD73 inhibitors APCP (A) for $3 \mathrm{~d}$. The production of IFN- $\gamma$ by CD $8^{+} \mathrm{T}$ cells were determined and summarized by flow cytometry $(\mathrm{n}=4)$. * indicates a $\mathrm{P}$ value of $<0.05$; ** indicates a $\mathrm{P}$ value of $<0.01$; *** indicates a $\mathrm{P}$ value of $<0.0001$. MDSCs, myeloid-derived suppressor cells; M-MDSCs, monocytic myeloid-derived suppressor cells; PMN-MDSCs, polymorphonuclear myeloid-derived suppressor cells; HNSCC, head and neck squamous cell carcinoma; APCP, adenosine 5 '-( $\alpha, \beta$-methylene)diphosphate; IFN- $\gamma$; interferon- $\gamma$.

production of MDSCs originated at the tumor site (34). Infiltration of the tumors by MDSCs was not determined in this study; because of the low number of cases at TNM stage II and TNM stage III, significant increases in MDSC levels may be detected using a larger sample of patients with later-stage HNSCC cancer.

Analysis of the percentages of PMN-MDSCs at different clinical cancer stages revealed that they were directly correlated. It is common for PMN-MDSCs to express CD15, while other markers, such as CD66b and IL4R $\alpha$, vary between different studies (35). Although direct evidence for the correlation between PMN-MDSCs and clinical characteristics is not strong, PNM-MDSCs play a crucial part in the progression of cancer (36). In renal carcinoma patients, PMN-MDSCs are significantly increased compared to in healthy donors (37). A subpopulation of PMN-MDSCs with reduced migration and oxidative burst has been identified in HNSCC (38). In metastatic colorectal cancer patients, levels of $\mathrm{T}$ regulatory cells, Th17, and PMN-MDSCs were significantly increased, but only high levels of PMN-MDSCs were associated with poor prognoses (39). These results indicate that PMN-MDSCs may represent an MDSC subpopulation with clinical signatures for cancer.

It has been observed that MDSCs can suppress cancer immunity using CD39 (19). The activity of CD73 is critical in immunosuppressive pathways in several cancer types, including HNSCC, thyroid, ovarian, and colorectal cancer $(23,25,29,30,39)$. In the heterogeneous population of MDSCs, CD73 expression displayed considerable heterogeneity. We found that $\mathrm{CD} 73$ was expressed at higher levels in M-MDSCs and PMN-MDSCs from HNSCC patients than those of healthy donors. The PMN-MDSCs had high level of CD73 and correlated with increasing clinical stages while no such correlation was observed between M-MDSCs and clinical stages. This observation is consistent with murine studies that high levels of CD73 are expressed in granulocytic-type MDSCs $(40,41)$. Increased percentages of PD-L1, CD39, and CD73 were found to be expressed in PMN-MDSCs from 25 metastatic colorectal cancer patients, which had a high level of immunosuppression compared to other myeloid cell types (39). Several groups $(24,29)$ reported CD73 expression in HNSCC by immunohistochemical analysis of tumor specimens that positively correlated with tumor stage and were associated with poor prognoses. Immunosuppression of $\mathrm{T}$ cell function by M-MDSCs and PMN-MDSCs is inhibited in an ectonucleotidase-dependent manner. Although both M-MDSCs and PMN-MDSCs immuno-suppress T cells, PMN-MDSCs exert a greater immunosuppressive 
effect. Similar results were obtained in an IFN- $\gamma$ ELISA assay. A greater effect was exerted by APCP on the immunosuppressive ability of PMN-MDSCs compared to M-MDSCs. Our results indicate that PMN-MDSCs play a greater role in immunosuppression than M-MDSCs.

Our data revealed that levels of MDSC are significantly higher in HNSCC patients than in cancer-free volunteers. Increasing PMN-MDSC levels correlated with increasing clinical stages, indicating that PMN-MDSCs are a clinically significant factor in the progression of HNSCC. Our results demonstrated that CD $73^{+} \mathrm{PMN}-\mathrm{MDSCs}$ contribute to $\mathrm{T}$ cell immune suppression in HNSCC patients. Ectonucleotidase inhibitors are promising drugs in the clinical development of immunotherapy of HNSCC.

\section{Acknowledgments}

We would like to express our thanks and appreciation to the team members of the multidisciplinary and specialist nursing teams of our departmental Head and Neck Cancer Center.

Funding: This study was supported by grants from the General Research Projects of Zhejiang Medical and Health Program (2016KYB049, 2018KY310).

\section{Footnote}

Reporting Checklist: The authors have completed the MDAR reporting checklist. Available at https://dx.doi. org/10.21037/atm-21-2589

Data Sharing Statement: Available at https://dx.doi. org/10.21037/atm-21-2589

Conflicts of Interest: All authors have completed the ICMJE uniform disclosure form (available at https://dx.doi. org/10.21037/atm-21-2589). The authors have no conflicts of interest to declare.

Ethical Statement: The authors are accountable for all aspects of the work in ensuring that questions related to the accuracy or integrity of any part of the work are appropriately investigated and resolved. The study was conducted in accordance with the Declaration of Helsinki (as revised in 2013). Approval of the study was granted by the Institutional Review Board of the Zhejiang Cancer Hospital (No. IRB-2015-215). Signed informed consent forms were provided by all participants.

Open Access Statement: This is an Open Access article distributed in accordance with the Creative Commons Attribution-NonCommercial-NoDerivs 4.0 International License (CC BY-NC-ND 4.0), which permits the noncommercial replication and distribution of the article with the strict proviso that no changes or edits are made and the original work is properly cited (including links to both the formal publication through the relevant DOI and the license). See: https://creativecommons.org/licenses/by-nc-nd/4.0/.

\section{References}

1. Siegel RL, Miller KD, Fuchs HE, et al. Cancer Statistics, 2021. CA Cancer J Clin 2021;71:7-33.

2. Bonner JA, Boggs DH. Treatment intensity in locoregionally advanced head and neck cancer: recent investigation leads to new questions. Transl Cancer Res 2019;8:S188-S194.

3. Argiris A, Karamouzis MV, Raben D, et al. Head and neck cancer. Lancet 2008;371:1695-709.

4. Jiang L, Guo L, Wang K, et al. Three therapeutic regimens based on induction chemotherapy in locally advanced squamous cell carcinoma of the head and neck: a single center experience. Transl Cancer Res 2020;9:901-9.

5. Zou W. Immunosuppressive networks in the tumour environment and their therapeutic relevance. Nat Rev Cancer 2005;5:263-74.

6. Rabinovich GA, Gabrilovich D, Sotomayor EM. Immunosuppressive strategies that are mediated by tumor cells. Annu Rev Immunol 2007;25:267-96.

7. Gabrilovich DI, Nagaraj S. Myeloid-derived suppressor cells as regulators of the immune system. Nat Rev Immunol 2009;9:162-74.

8. Gabitass RF, Annels NE, Stocken DD, et al. Elevated myeloid-derived suppressor cells in pancreatic, esophageal and gastric cancer are an independent prognostic factor and are associated with significant elevation of the Th2 cytokine interleukin-13. Cancer Immunol Immunother 2011;60:1419-30.

9. Diaz-Montero CM, Salem ML, Nishimura MI, et al. Increased circulating myeloid-derived suppressor cells correlate with clinical cancer stage, metastatic tumor burden, and doxorubicin-cyclophosphamide chemotherapy. Cancer Immunol Immunother 2009;58:49-59.

10. Almand B, Clark JI, Nikitina E, et al. Increased production of immature myeloid cells in cancer patients: a mechanism of 
immunosuppression in cancer. J Immunol 2001;166:678-89.

11. Peranzoni E, Zilio S, Marigo I, et al. Myeloid-derived suppressor cell heterogeneity and subset definition. Curr Opin Immunol 2010;22:238-44.

12. Eruslanov E, Neuberger M, Daurkin I, et al. Circulating and tumor-infiltrating myeloid cell subsets in patients with bladder cancer. Int J Cancer 2012;130:1109-19.

13. Zhang B, Wang Z, Wu L, et al. Circulating and tumorinfiltrating myeloid-derived suppressor cells in patients with colorectal carcinoma. PLoS One 2013;8:e57114.

14. Brusa D, Simone M, Gontero P, et al. Circulating immunosuppressive cells of prostate cancer patients before and after radical prostatectomy: profile comparison. Int J Urol 2013;20:971-8.

15. Geissler K, Fornara P, Lautenschläger C, et al. Immune signature of tumor infiltrating immune cells in renal cancer. Oncoimmunology 2015;4:e985082.

16. Ostrand-Rosenberg S, Fenselau C. Myeloid-Derived Suppressor Cells: Immune-Suppressive Cells That Impair Antitumor Immunity and Are Sculpted by Their Environment. J Immunol 2018;200:422-31.

17. Bono MR, Fernández D, Flores-Santibáñez F, et al. CD73 and CD39 ectonucleotidases in T cell differentiation: Beyond immunosuppression. FEBS Lett 2015;589:3454-60.

18. Peng H, Xue R, Ju Z, et al. Cancer-associated fibroblasts enhance the chemoresistance of CD73+ hepatocellular carcinoma cancer cells via HGF-Met-ERK1/2 pathway. Ann Transl Med 2020;8:856.

19. Antonioli L, Yegutkin GG, Pacher P, et al. Anti-CD73 in cancer immunotherapy: awakening new opportunities. Trends Cancer 2016;2:95-109.

20. Allard B, Beavis PA, Darcy PK, et al. Immunosuppressive activities of adenosine in cancer. Curr Opin Pharmacol 2016;29:7-16.

21. Mandapathil M, Szczepanski MJ, Szajnik M, et al. Increased ectonucleotidase expression and activity in regulatory $T$ cells of patients with head and neck cancer. Clin Cancer Res 2009;15:6348-57.

22. Leclerc BG, Charlebois R, Chouinard G, et al. CD73 Expression Is an Independent Prognostic Factor in Prostate Cancer. Clin Cancer Res 2016;22:158-66.

23. Li L, Wang L, Li J, et al. Metformin-Induced Reduction of CD39 and CD73 Blocks Myeloid-Derived Suppressor Cell Activity in Patients with Ovarian Cancer. Cancer Res 2018;78:1779-91.

24. Ren ZH, Yuan YX, Ji T, et al. CD73 as a novel marker for poor prognosis of oral squamous cell carcinoma. Oncol
Lett 2016;12:556-62.

25. Mandapathil M, Boduc M, Netzer C, et al. CD73 expression in lymph node metastases in patients with head and neck cancer. Acta Otolaryngol (Stockh) 2018;138:180-4.

26. Li J, Wang L, Chen X, et al. CD39/CD73 upregulation on myeloid-derived suppressor cells via TGF- $\beta$-mTORHIF-1 signaling in patients with non-small cell lung cancer. Oncoimmunology 2017;6:e1320011.

27. Haun RS, Quick CM, Siegel ER, et al. Bioorthogonal labeling cell-surface proteins expressed in pancreatic cancer cells to identify potential diagnostic/therapeutic biomarkers. Cancer Biol Ther 2015;16:1557-65.

28. Yu YI, Wang W, Song L, et al. Ecto-5'-nucleotidase expression is associated with the progression of renal cell carcinoma. Oncol Lett 2015;9:2485-94.

29. Kondo T, Nakazawa T, Murata SI, et al. Expression of CD73 and its ecto-5'-nucleotidase activity are elevated in papillary thyroid carcinomas. Histopathology 2006;48:612-4.

30. Wu XR, He XS, Chen YF, et al. High expression of CD73 as a poor prognostic biomarker in human colorectal cancer. J Surg Oncol 2012;106:130-7.

31. Koontongkaew S. The tumor microenvironment contribution to development, growth, invasion and metastasis of head and neck squamous cell carcinomas. J Cancer 2013;4:66-83.

32. Weed DT, Vella JL, Reis IM, et al. Tadalafil reduces myeloid-derived suppressor cells and regulatory $T$ cells and promotes tumor immunity in patients with head and neck squamous cell carcinoma. Clin Cancer Res 2015;21:39-48.

33. Chikamatsu K, Sakakura K, Toyoda M, et al. Immunosuppressive activity of CD14+ HLA-DR-cells in squamous cell carcinoma of the head and neck. Cancer Sci 2012;103:976-83.

34. Maenhout SK, Thielemans K, Aerts JL. Location, location, location: functional and phenotypic heterogeneity between tumor-infiltrating and non-infiltrating myeloid-derived suppressor cells. Oncoimmunology 2014;3:e956579.

35. Dumitru CA, Moses K, Trellakis S, et al. Neutrophils and granulocytic myeloid-derived suppressor cells: immunophenotyping, cell biology and clinical relevance in human oncology. Cancer Immunol Immunother 2012;61:1155-67.

36. Kramer ED, Abrams SI. Granulocytic Myeloid-Derived Suppressor Cells as Negative Regulators of Anticancer Immunity. Front Immunol 2020;11:1963. 
37. Rodriguez PC, Ernstoff MS, Hernandez C, et al. Arginase I-producing myeloid-derived suppressor cells in renal cell carcinoma are a subpopulation of activated granulocytes. Cancer Res 2009;69:1553-60.

38. Brandau S, Trellakis S, Bruderek K, et al. Myeloid-derived suppressor cells in the peripheral blood of cancer patients contain a subset of immature neutrophils with impaired migratory properties. J Leukoc Biol 2011;89:311-7.

39. Limagne E, Euvrard R, Thibaudin M, et al. Accumulation of MDSC and Th17 Cells in Patients with Metastatic Colorectal Cancer Predicts the Efficacy of a FOLFOX-

Cite this article as: Zheng W, Zhu Y, Chen X, Zhao J. CD73 expression in myeloid-derived suppressor cells is correlated with clinical stages in head and neck squamous cell carcinomas. Ann Transl Med 2021;9(14):1148. doi: 10.21037/atm-21-2589
Bevacizumab Drug Treatment Regimen. Cancer Res 2016;76:5241-52.

40. Ryzhov S, Novitskiy SV, Goldstein AE, et al. Adenosinergic Regulation of the Expansion and Immunosuppressive Activity of CD11b + Gr1 + Cells. J Immunol 2011;187:6120-9.

41. Ryzhov SV, Pickup MW, Chytil A, et al. Role of TGF- $\beta$ signaling in generation of CD39+CD73+ myeloid cells in tumors. J Immunol 2014;193:3155-64.

(English Language Editor: J. Jones) 